\title{
A Low-Noise, Non-Contact EEG/ECG Sensor
}

\author{
Thomas J. Sullivan, Stephen R. Deiss, and Gert Cauwenberghs \\ Division of Biological Sciences, University of California, San Diego \\ La Jolla, CA 92093-0368 USA \\ Email: tom@sullivan.to,sdeiss@ucsd.edu,gert@ucsd.edu
}

\begin{abstract}
Typical electroencephalogram (EEG) and electrocardiogram (ECG) sensors require conductive gel to ensure low-impedance electrical contact between the sensor and skin, making set-up time-consuming and long-term recording problematic. We present a gel-free, non-contact EEG/ECG sensor with on-board electrode that capacitively couples to the skin. Active shielding of the high-impedance input significantly reduces noise pickup, and reduces variations in gain as a function of gap distance. The integrated sensor combines amplification, bandpass filtering, and analog-to-digital conversion within a 1 inch diameter enclosure. The measured input-referred noise, over $1-100 \mathrm{~Hz}$ frequency range, is $2 \mu \mathrm{Vrms}$ at $0.2 \mathrm{~mm}$ sensor distance, and $17 \mu$ Vrms at $3.2 \mathrm{~mm}$ distance. Experiments coupling the sensor to human scalp through hair and to chest through clothing produce clear EEG and ECG recorded signals.
\end{abstract}

Index Terms-capacitive sensing, non-contact biopotential sensor, EEG, ECG.

\section{INTRODUCTION}

$\mathrm{E}$ EG SENSORS measure the time-varying magnitude of electric fields emanating from the brain. These fields result from the collective synchronous activity of large numbers of neurons. Typical EEG sensors record electrical potentials measuring these fields on the scalp. Their precise measurement relies on direct, low-resistance contact between the sensor and scalp. This is usually accomplished by applying an electrically-conductive gel between the sensor and scalp.

While the gel achieves the aim of making a good contact, several problems result. It takes up to an hour to apply the gel into EEG caps that use 256 sensors. The gel may diffuse through the hair to create shorts between sensors. It may also dry out over time, making long term recordings very difficult. Our non-contact sensor avoids ohmic contact with skin altogether and operates by capacitive coupling, capable of measuring EEG signals through hair, or ECG signals through clothing.

There have been many attempts to use sensors that do not require gel, but still rely on dry contact with the skin [1], [2]. These approaches are limited to body areas with no hair. Early non-contact, electric field sensors for biomedical use were reportedly successful in reading electric fields produced by the heart (an electrocardiogram or ECG) [3] as well as EEG [4]. Modern integrated circuits and fabrication techniques are used here to improve the performance and manufacturability of this type of sensor. Prance and others [5], [6] used low input-bias current amplifiers that yield lownoise operation at low frequencies. Further advances in noncontact ECG sensors were reported by Matthews and others [7], [8]. In this contribution we report a compact, low-noise EEG/ECG sensor with integrated digital readout, and include detailed circuit schematics and measured performance.

\section{SENSOR DESIGN}

\section{A. Circuit Design}

The circuit that senses, amplifies and acquires the signal is shown in Fig. 1. The signal on the skin capacitively couples to a metal plate at the bottom of the printed circuit board (PCB), which is covered with solder mask for electrical insulation of the sensor.

First amplification of the signal is accomplished by a INA116 instrumentation amplifier, configured for a gain of 50. This component has an extremely low input bias current of $3 \mathrm{fA}$ (typical) and an input current noise of $0.1 \mathrm{fA} / \sqrt{\mathrm{Hz}}$ (typical). It also features guard pin outputs, which follow the positive and negative inputs with a gain of 1 . In addition to using the positive guard to support a guard ring around the positive input pin, we also use it to drive a shielding metal plate that minimizes electric field pick up from sources other than the scalp, as in [5]. This shield is implemented as an inner layer of metal on the PCB above the sensor metal layer. Because it is actively driven to duplicate the input voltage, it avoids parasitic capacitance division of signal gain.

The input bias current is extremely small, but if left unattended will drive the high-impedance positive input node of the amplifier toward one of the supply rails. We combat this with a reset circuit which includes two transistors and two resistors. The transistors are turned on by an external circuit when the input voltage nears the common-mode input range of the amplifier. When not driven, the base and emitter nodes of the transistors are pulled up by the guard output. This is done to minimize leakage currents (and especially the resultant current noise) from the transistors. The negative amplifier input is made to track the slowly changing positive input with the feedback loop consisting of R4 and C4. This loop also serves to cut off input signals of frequencies below 1 $\mathrm{Hz}$. 


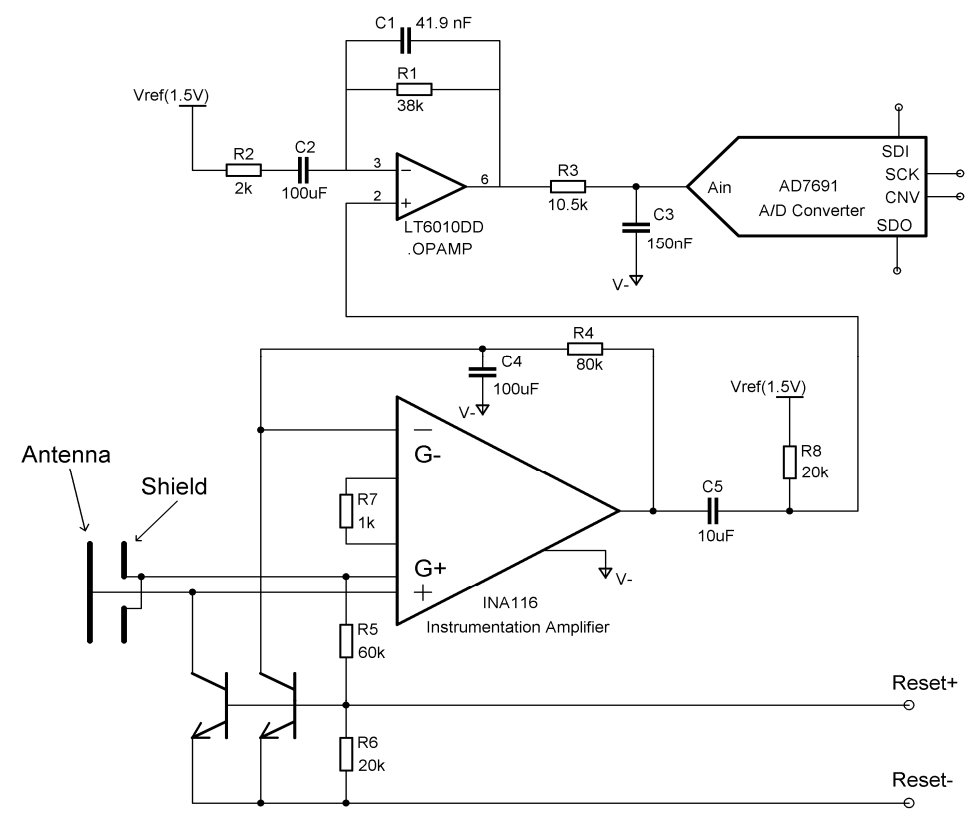

Fig. 1. Circuit schematic. The EEG/ECG signal on the skin capacitively couples to a metal plate on the bottom of the PCB. The two amplifiers combine to create a signal gain of 1000. The feedback loop created by R4 and C4 serve to make the negative input terminal of the first amplifier track the slowly changing positive input terminal. When the input voltage is in danger of going out of range, the reset terminals are used to control the transistors and reset the input nodes of the amplifier. The 18-bit ADC converts the signal to a stream of bits.
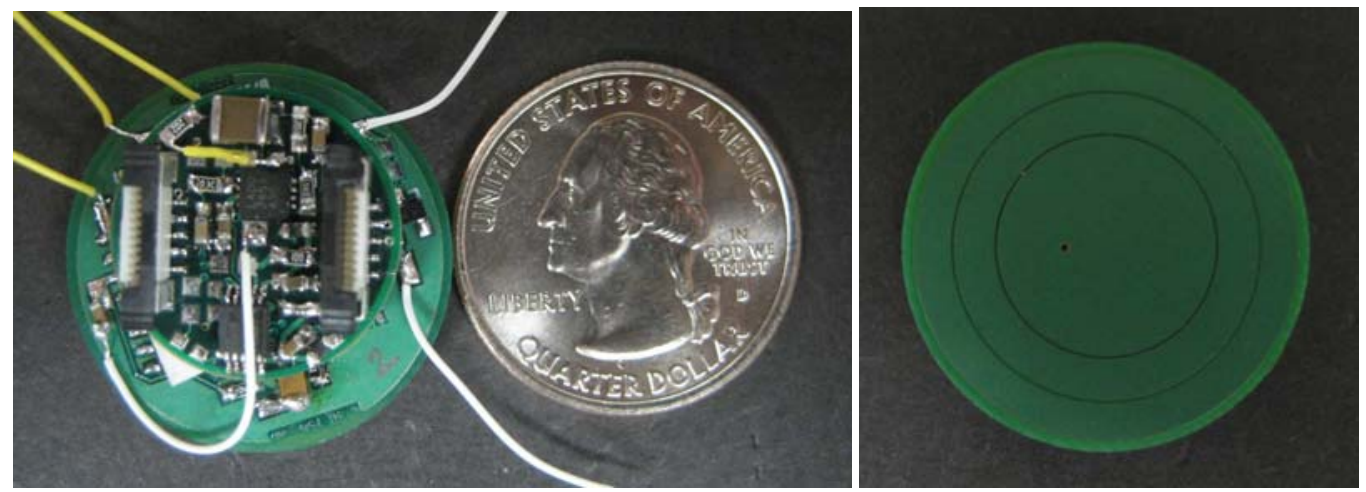

Fig. 2. Photograph of assembled circuit. (Left). The top side of the two-board structure with components. (Right). The bottom side of the bottom PCB. The metal plate on the bottom is the sensing element, which is covered by solder mask.
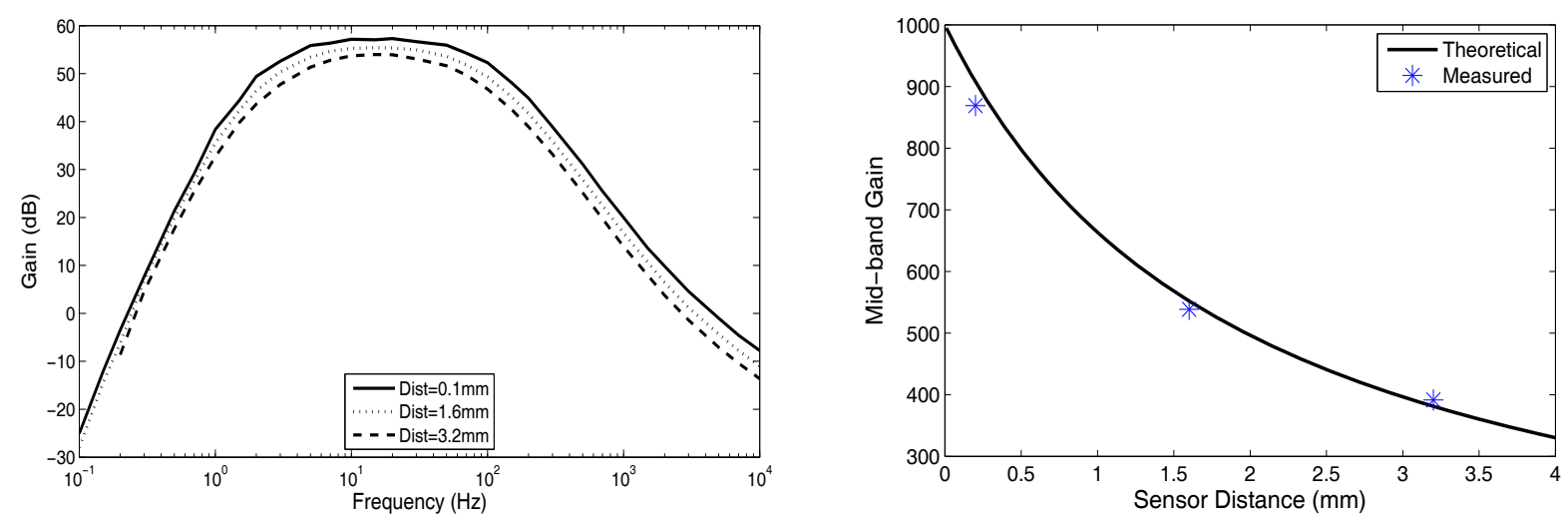

Fig. 3. Effect of sensor separation distance on signal gain. (Left) The filtering sets up a bandpass characteristic between 1 and $100 \mathrm{~Hz}$. The gain versus frequency for three distances between sensor and signal generator are shown. The overall gain decreases as the distance increases. This is due to the capacitive signal divider formed at the input. (Right) The measured mid-band gain at three sensor separation distances agree well with theory. 

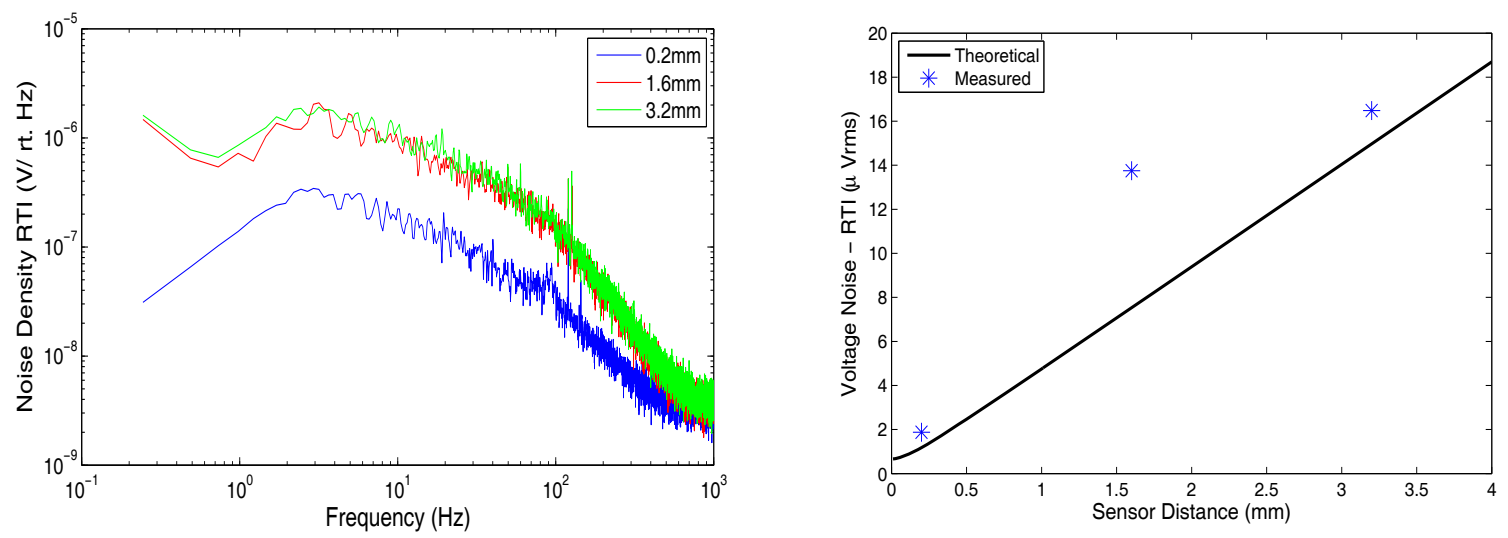

Fig. 4. Effect of sensor separation distance on input-referred noise. (Left) Noise density over frequency. With the input generating plate grounded and a sensor distance of $0.2 \mathrm{~mm}, 1.6 \mathrm{~mm}$, and $3.2 \mathrm{~mm}$ a spectral density estimate was taken at the output. Output noise was referred to the input by dividing by the midband gain in Fig. 3. (Right) Noise as a function of sensor distance. The input-referred rms noise is measured over the 1-100 $\mathrm{Hz}$ frequency band for the three sensor separation distances, and compared with the theoretically expected noise.

The output of the instrumentation amplifier is level-shifted and sent to an LT6010. This operational amplifier is configured for a gain of 20 . A zero is inserted at $1 \mathrm{~Hz}$ by $\mathrm{C} 2$ to further cut off signals below that frequency. Two poles are implemented at $100 \mathrm{~Hz}$ by $\mathrm{C} 1$ reacting with $\mathrm{R} 1$ and $\mathrm{C} 3$ with R3. This completes the bandpass filter characteristic between $1 \mathrm{~Hz}$ and $100 \mathrm{~Hz}$.

An AD7691 18-bit analog-to-digital converter (ADC) converts the signal to a stream of digital bits at a rate of $2 \mathrm{kS} / \mathrm{s}$ [10]. The interface can be daisy-chained with other sensor ADCs to decrease the number of wires in the system. The output of the $\mathrm{ADC}$ was connected to a data acquisition card on a desktop PC for device characterization.

The total current needed for the INA116 is $1 \mathrm{~mA}$ from $+5 \mathrm{~V}$ and $-5 \mathrm{~V}$ power supplies. The LT6010 and AD7691 use a single-ended $3 \mathrm{~V}$ supply and require $160 \mu \mathrm{A}$ total. The total power required is $10.5 \mathrm{~mW}$, which means that a hundred sensors can run for hours on a battery pack.

\section{B. Layout}

Fig. 2 shows photographs of the circuit implementation. Two custom PCBs were designed, assembled, and stacked one upon the other. The top board contains the operational amplifier, A-to-D converter and some passive components. The bottom board has the sensor's metallic plate, shielding plate, instrumentation amplifier and transistors. The complete system is shown on the left, next to a U.S. quarter for size comparison. The photo on the right shows the bottom of the bottom board. The bottom layer of the PCB is all metal covered with solder mask.

\section{CHARACTERIZATION}

\section{A. Gain and Phase}

The gain and phase of the circuit were tested with a lock-in amplifier. The input signal was applied to a metal plate that was separated from the sensor at variable distance. The smallest distance is that created by just the soldermask, which is estimated to be $0.2 \mathrm{~mm}$. The two larger distances are formed with spacers made of an acrylic plastic. Its dielectric constant of about 4 is roughly the same as that of human hair [9]. The output was taken at the input of the ADC.

Fig. 3 (left panel) shows the measured gain of the sensor over a range of frequencies. The bandpass characteristic of the filtering between $1 \mathrm{~Hz}$ and $100 \mathrm{~Hz}$ is evident. The $1 \mathrm{~Hz}$ cutoff is steeper as there are three zeros acting there caused by the feedback loop of the instrumentation amplifier, $\mathrm{C} 2$ in the second amplifier feedback, and the level-shifter formed by C5 and R8. The two poles discussed previously act at $100 \mathrm{~Hz}$.

The EEG input can be modeled as a voltage source coupled into the circuit through a capacitor. The capacitance is calculated as the area of the sensing plate divided by the distance between the sensor and scalp. Since there is also parasitic capacitance on the positive input node of the instrumentation amplifier a capacitive voltage divider is formed at the input which reduces the signal strength. Fig. 3 (right panel) shows the gain for three different distances between the signal generator and the sensor. As the distance is increased, the input coupling capacitance is reduced, as is the overall gain of the circuit. At a distance of $0.2 \mathrm{~mm}$, the gain is 869 , whereas it is 539 at $1.6 \mathrm{~mm}$ and 391 at $3.2 \mathrm{~mm}$. The reduction in gain with distance is significantly larger when the active shield is replaced with a passive ground shield. With active shield, the EEG/ECG sensor is capable to operate over a wide range of distances as encountered with typical hair and clothing between sensor and skin.

\section{B. Noise}

Perhaps the most difficult challenge of EEG sensor design is in creating an amplifier circuit with very low noise. The signals being measured can reach down to tens of microvolts peak-to-peak so noise levels below this are desirable. In our circuit, the ADC is not a significant source of noise since it converts a signal that has already seen a large gain and it converts at 18-bit levels. The second amplifier also does not contribute significant noise since it comes after the initial gain of 50. The calculated referred-to-input (RTI) voltage noise of the amplifier in the frequency band from 1 to $100 \mathrm{~Hz}$ is about 
$0.66 \mu \mathrm{Vrms}$. The RTI current noise of the instrumentation amplifier, though extremely small, is integrated by the capacitance seen at the positive input terminal. Assuming a sensor distance of $0.2 \mathrm{~mm}$ this current noise is converted to about $1 \mu$ Vrms. Ideally, the guard input will keep the transistor terminals all at the same voltage, keeping their leakage noise currents near zero. The resistor R4, though large, produces thermal noise that is reduced by the feedback loop and is not a significant factor. So, in total, the expected RTI voltage noise is under $2.0 \mu \mathrm{Vrms}$. The measured noise density as a function of frequency is shown in Fig. 4 (left panel). This leads to a measured noise of $1.88 \mu \mathrm{Vrms}$.

As the sensor separation distance increases, the coupling capacitance decreases. The current noise is then integrated to a larger voltage noise value. The theoretically calculated noise is shown in Fig. 4 (right panel) along with measurements at the same three distances used for the gain measurements. The theoretical curve accounts for the amplifier's current and voltage input-referred noise, and for the capacitive division at the input.

\section{EEG/ECG Data}

Human physiology data was taken in a typical electrical engineering laboratory in order to verify the effectiveness of the sensors in a real-world setting. First, two sensors were pressed against a subject's head using a headband. The first sensor was located in the back of the head (on top of the hair), while the second was located behind the ear and used as a reference. The voltage difference between the two was recorded as the subject first closed his eyes for 12 seconds then kept them open for the same amount of time. The power spectral densities of the data from these two blocks of time are shown in Fig. 5. Increased power in the alpha band of frequencies around $10 \mathrm{~Hz}$ can clearly be seen when the eyes are closed, commonly observed in EEG experiments.

The second experiment also employed two sensors. One was located on top of the chest near the heart, the second was located on the side of the chest and used as a reference. Both were placed outside the subject's t-shirt. The difference between the two sensors is shown in Fig. 6. A signal caused by the beating heart is clearly visible.

\section{DISCUSSION}

Non-contact electric field sensors have the potential to revolutionize biopotential recording systems and humanmachine interfaces. We have presented a low-noise integrated sensor for EEG and ECG signals that operates up to $3 \mathrm{~mm}$ distance from the skin, and produces bit-serial digital readout for daisy-chain operation over large numbers of sensors.

Our versatile sensor can be applied in many biopotential applications. In the future, we intend to show its use in an EMG application which would only require a widening of the bandpass filter characteristic to include signals up to $1000 \mathrm{~Hz}$.

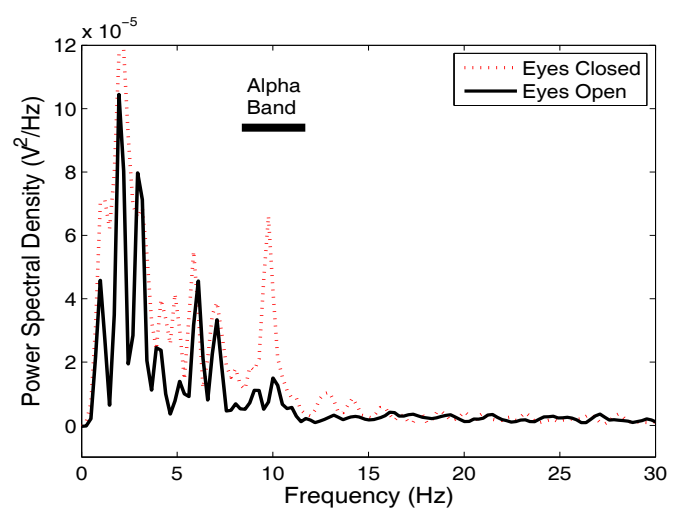

Fig. 5. Alpha waves. One sensor was place on the back of the subject's head and one was placed behind the ear as a reference. The subject closed eyes for 12 seconds and kept them open for 12 seconds. The power spectrum of the two blocks of data reveals that the power near 10 $\mathrm{Hz}$, commonly called alpha waves, is bigger with the eyes closed. This is commonly observed in EEG research.

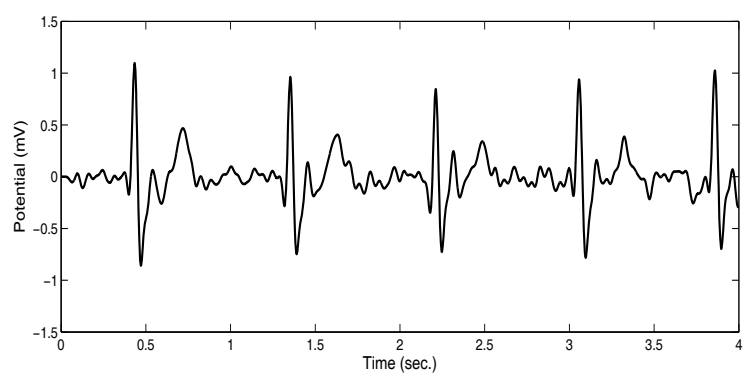

Fig. 6. ECG. A typical ECG voltage was measured through a T-shirt. Shown is the potential difference between two sensors near the heart.

\section{REFERENCES}

[1] iCap Technologies, http://www.icaptech.com/.

[2] J. C. Chiou, Li-Wei Ko, Chin-Teng Lin, Chao-Ting Hong, Tzyy-Ping Jung, "Using Novel MEMS EEG Sensors in Detecting Drowsiness Application," IEEE Biomedical Circuits and Systems Conference, 2006.

[3] A. Lopez and P. C. Richardson, "Capacitive electrocardiographic and bioelectric electrodes", IEEE Transactions on Biomedical Engineering, vol. 16, pg. 99, 1969.

[4] T. Matsuo, K. Iinuma, and M. Esashi, "A barium-titanate-ceramics capacitive-type EEG electrode", IEEE Transactions on Biomedical Engineering, vol. 188, pgs 299-300.

[5] R. J. Prance, A. Debray, T. D. Clark, H. Prance, M. Nock, C. J. Harland, and A. J. Clippingdale, "An ultra-low-noise electrical-potential probe for human-body scanning", Measurement Science and Technology, vol. 11, pgs. $291-297,2000$.

[6] C. J. Harland, T. D. Clark and R. J. Prance, "Electric potential probesnew directions in the remote sensing of the human body", Measurement Science and Technology, vol. 13, pgs. 163-169, 2002.

[7] R. Matthews, N. J. McDonald, I. Fridman, P, Hervieux, and T. Nielsen, "The invisible electrode - zero prep time, ultra low capacitive sensing. In Proceedings of the $11^{\text {th }}$ International Conference on HumanComputer Interaction, July 22-27 2005.

[8] C. Park, P. H. Chou, Y. Bai, R. Matthews, and A. Hibbs, "An ultrawearable, wireless, low power ECG monitoring system", IEEE Biomedical Circuits and Systems Conference, 2006.

[9] J. Errera and H. S. Sack, "Dielectric properties of animal fibers"

[10] T. Sullivan, S. Deiss, T.P. Jung, and G. Cauwenberghs, "A Low-Noise, Low-Power EEG Acquisition Node for Scalable Brain-Machine Interfaces", In Proceedings of the SPIE Conference on Bioengineered and Bioinspired Systems III, May 2-4 2007. 